\title{
THE RADIAL DISTRIBUTION OF GAMMA RAYS AND COSMIC RAYS IN THE OUTER GALAXY ${ }^{\dagger}$
}

\author{
W. Hermsen, ${ }^{*}$ L. Blitz****** and \\ J. B. G. M. Bloemen ${ }^{*, * * *}$ \\ ${ }^{*}$ Cosmic-Ray Working Group, Huygens Laboratorium, \\ Leiden, The Netherlands \\ *: Sterrewacht, Huygens Laboratorium, Leiden, The Netherlands \\ *:*: Astronomy Program, University of Maryland, MD, U.S.A.
}

\section{ABSTRACT}

The radial distribution of the high-energy ( $70 \mathrm{MeV}-5 \mathrm{GeV}$ ) gamma-ray emissivity in the outer Milky way is derived. The kinematics of HI are used to construct column-density maps in three galacto-centric distance ranges in the outer Galaxy. These maps are used in combination with COS-B gamma-ray data to determine gamma-ray emissivities in these distance ranges. A steep negative gradient of the emissivity for the $70 \mathrm{MeV}-150 \mathrm{MeV}$ energy range is found in the outer Galaxy. The emissivity for the $300 \mathrm{MeV}-5 \mathrm{GeV}$ range is found to be approximately constant (within $220 \%$ ) and equal to the local value out to large ( $20 \mathrm{kpc}$ ) galacto-centric distances. These results imply a hardening of the gamma-ray spectrum with increasing distance and for $\mathrm{R}>16 \mathrm{kpc}$ the spectrum is shown to be consistent with a $\pi^{\circ}$-decay spectrum with the intensity expected from the local measurement of the cosmic-ray nuclei spectrum. The energy-dependent decrease is interpreted as a steep gradient in the cosmic-ray electron density and a near constancy of the nuclear component. The galactic origin of electrons with energies up to several hundreds of $\mathrm{MeV}$ is confirmed, while for cosmic-ray nuclei with energies of a few GeV either confinement in a large galactic halo or an extragalactic origin is suggested by the data.

\section{KEYWORDS}

Cosmic rays; gamma rays; interstellar matter; COS-B

\section{INTRODUCTION}

The diffuse component of galactic high-energy ( $\lesssim 35 \mathrm{MeV}$ ) gamma rays has long been interpreted to be mainly the result of the interaction of cosmic-ray electrons (via bremsstrahlung) and cosmic-ray nuclei (via $\pi^{\circ}$-decay) with the interstellar gas. To date these two contributions have not been separated observationally despite their different spectral shapes. Various authors have shown that the major part of the observed galactic gamma-ray emission can be explained by the cosmic-ray-matter interactions (e.g. Strong et al. 1982 and Lebrun et $\alpha l$. 1983). While in the inner Galaxy the contribution from cosmic-ray interactions with molecular gas is significant, for the outer Galaxy Bloemen, Blitz and Hermsen (1983) have shown that the gamma-ray intensity is proportional to the HI column density alone within the uncertainties of the analysis. From the radial distribution of the HI, with which the gamma rays are correlated, they conclude that a significant fraction of the galactic gamma-ray emission originates at large galacto-centric distances and that $250 \%$ of the total gamma-ray 1 uminosity of the Galaxy is produced beyond the solar circle.

Both, from SAS-2 data (Dodds et al. 1975, Issa et al. 1980 and Wolfendale 1981) and from COS-B data (Mayer-Hasselwander et al. 1982) indications were found for a lower emissivity in the outer Galaxy compared to the local value. Bloemen, Blitz and Hermsen (1983) showed, using Cos-B data, that this decrease might on 1y be present for low-energy ( $70 \mathrm{MeV}-150 \mathrm{MeV}$ ) gamma rays. In this paper, the gamma-ray emissivity spectrum is derived for three distance ranges in the second and third galactic quadrants and is discussed in terms of the contribution from bremsstrahlung and $\pi^{\circ}$-decay and of the distribution of cosmic rays.

\section{RADIAL DISTRIBUTIONS OF GAMMA-RAY EMISSIVITIES}

Since $H_{2}$ can be neglected for $R>R_{0}$ (in this paper we take $R_{g}=10 \mathrm{kpc}$ ) the velocity information from the $21-\mathrm{cm}$ line can be used to determine the spatial distribution of the $H I$ for $R>R_{\odot}$. We used the rotation curve of the outer Galaxy given by Blitz, Fich and Stark (1980) as modified by Kulkarni, Blitz and Heiles (1982) to determine distances beyond the solar circle. Using

\footnotetext{
Ton behalf of the Caravane Collaboration
} 


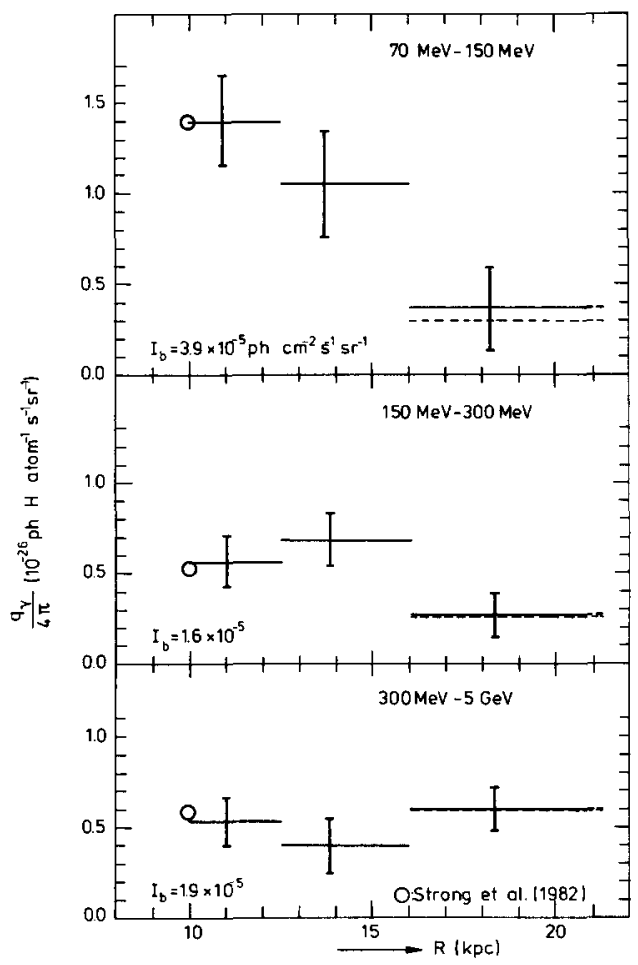

Fig. 1. Radial distribution of $\mathrm{q}_{\gamma}$ in the outer Galaxy for three energy ranges. the resulting isotropic background levels $\mathrm{I}_{\mathrm{b}}$ are given in the figures. The errorbars indicate formal $1 \sigma$ errors. The dashed 1 ines for $\mathrm{R}>16 \mathrm{kpc}$ show the values of $q_{\gamma}$ after correction for a $\pi^{\circ}$-decay input spectrum.

this distance information, HI column densities can be determined in various galacto-centric distance ranges and their contribution to the observed gamma-ray intensities can be investigated. The analysis was performed in the longitude range covered by the HI surveys of Weaver and Williams (1973) and Heiles and Habing (1974) in the second and third galactic quadrants. Those regions where contamination from point-1ike gamma-ray sources (Swanenburg et al. 1981) and known concentrations of molecules between $Z \cong 107^{\circ}$ and $l \cong 113^{\circ}$ might affect the analysis were excluded (a posteriori it was verified that inclusion of these regions would not have changed the conclusions reached in this paper). The remaining data were analyzed for $95^{\circ}<Z<245^{\circ}$ and $|\beta|<10^{\circ}$. Gamma-ray intensity maps in three energy ranges ( $70 \mathrm{MeV}-150 \mathrm{MeV}, 150 \mathrm{MeV}-300 \mathrm{MeV}$ and $300 \mathrm{MeV}-5 \mathrm{GeV}$ ) were derived from the $\operatorname{COS}-\mathrm{B}$ data base, assuming an $\mathrm{E}^{-2}$ input spectrum in order to take into account the energy dependence of the instrument response. The impact of this assumption on the results was evaluated a posteriori and, where significant, the results have been corrected. The HI surveys were corrected to obtain the brightness temperature $T_{b}$ (Williams, 1973), and a first order optical-depth correction was made assuming an uniform spin temperature of $125 \mathrm{~K}$. HI column-density maps have been constructed for the gas in three distance intervals: $R<12.5 \mathrm{kpc}, 12.5 \mathrm{kpc}<\mathrm{R}<16 \mathrm{kpc}$ and $\mathrm{R}>16 \mathrm{kpc}$. The distance intervals were chosen so that comparable fractions of the total average HI column density are contained in each interva1. The maps have been convolved with the COS-B point-spread functions (Hermsen, 1980) for the energy ranges to be analyzed. Because of the large scale warp of the hydrogen layer, which is more pronounced for increasing galacto-centric distances (see e.g. Henderson, Jackson and Kerr 1982 and Kulkarni, Blitz and Heiles 1982), the distributions of N(HI) in the three distance ranges are quite different.

Because of this difference in the projected distributions it can be investigated which combination of gamma-ray emissivities inside $12.5 \mathrm{kpc}\left(\mathrm{q}_{\gamma, 1}\right)$, between $12.5 \mathrm{kpc}$ and $16 \mathrm{kpc}\left(\mathrm{q}_{\gamma, 2}\right)$ and outside $16 \mathrm{kpc}\left(\mathrm{q}_{\gamma},{ }^{3}\right)$ best describes the observed gamma-ray distribution, using a relation of the form:

$$
I_{\gamma}=\frac{1}{4 \pi}\left\{q_{\gamma, 1} \tilde{N}(H I)_{1}+q_{\gamma, 2} \tilde{N}(H I)_{2}+q_{\gamma, 3} \tilde{N}(H I)_{3}\right\}+I_{b}
$$

where $\tilde{H}(\mathrm{HI})_{1}, \tilde{\mathrm{N}}(\mathrm{HI})_{2}$ and $\tilde{\mathrm{N}}(\mathrm{HI})_{3}$ are the convolved $\mathrm{HI}$ column densities in the three distance ranges. $I_{b}$ represents the total isotropic background (mainly instrumental). A maximum-1ikelihood method, similar to that used by Lebrun et $\alpha \mathrm{L}$. (1982), was applied on $1^{\circ} \times 1^{\circ}$ bins to determine $q_{\gamma, 1}, q_{\gamma, 2}, q_{\gamma, 3}$ and $I_{b}$ for each energy range. The resulting $f i t$ values, presented in Fig. 1, show that the emissivity decreases with increasing $\mathrm{R}$ for the $70 \mathrm{MeV}-150 \mathrm{MeV}$ range, while the emissivity for higher energies (150 MeV-5 GeV) remains approximately constant 


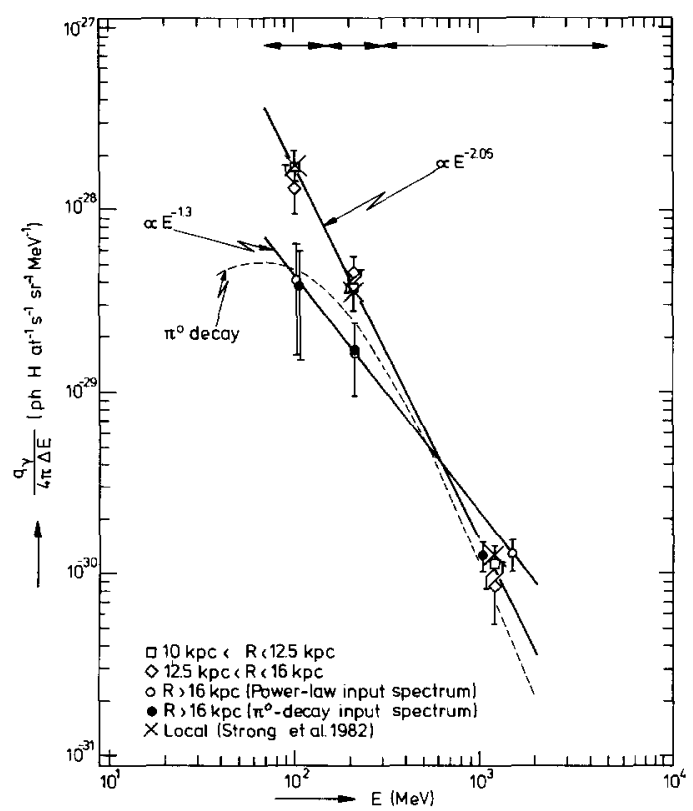

Fig. 2. The gamma-ray emissivity spectrum for three distance ranges in the outer Galaxy. Formal $(1 \sigma)$ errorbars are indicated. The energy ranges in which the emissivities are derived, are given at the top of the figure. The solid lines indicate the best power-law fits for $R<12.5 \mathrm{kpc}$ and $\mathrm{R}>16 \mathrm{kpc}$. The dashed curve shows the $\pi^{\circ}$-decay spectrum of Stephens and Badwahr (1981) (lowered by $10 \%$ to $f$ it better the data points) determined from the demodulated proton spectrum given by Burger and Swanenburg (1971).

(within 20\%) out to large distances. Various checks were made on the significance of the gradients found (see Bloemen et al., 1984). For the $70 \mathrm{MeV}-150 \mathrm{MeV}$ range the likelihood was found to reduce by a factor of about 10 when a constant emissivity is assumed.

Figure 2 presents the gamma-ray emissivity spectrum for the three distance ranges. There is a clear hardening of the spectrum for increasing galacto-centric distances outside the solar circle. The best power-law fits are indicated in the figure. For $R>16 \mathrm{kpc}$, the spectrum is equally well fitted by a $\pi^{\circ}$-decay spectrum and is thus the first measurement of a diffuse gamma-ray spectrum consistent with the $\pi^{0}$-decay spectrum.

\section{RADIAL DISTRIBUTIONS OF COSMIC-RAY ELECTRONS AND NUCLEI}

The knowledge of the radial distribution of gamma-ray emissivities for different energy ranges enables the determination of the radial distribution of cosmic ray electrons and protons seperately, assuming that cosmic-ray-matter interactions are responsible for the observed gamma-ray emission. The inverse Compton contribution is most probably negligible for the outer Galaxy (see e.g. Kniffen and Fichtel (1981) and Sacher and Schönfelder (1983)).

The overall shape of the local gamma-ray emissivity spectrum indicates that gamma-ray emission in the $70 \mathrm{MeV}-150 \mathrm{MeV}$ range is predominantly due to bremsstrahlung of electrons (with Ex300 $\mathrm{MeV}$ ). The detected decrease of the gamma-ray emissivity for the low-energy range therefore implies that there is an evident galacto-centric gradient in the cosmic-ray electron density for electrons with $\hat{\mathrm{E}}\{300 \mathrm{MeV}$. It is reasonable to assume that a similar decrease occurs in the higher-energy electron distribution. Since there is no detectable gradient for the highenergy gamma-ray emissivities, interactions of cosmic-ray nucleons with the gas must dominate at these energies and the nucleon density must be constant out to large distances. This is supported by the close agreement between the measured $300 \mathrm{MeV}-5 \mathrm{GeV}$ gamma-ray emissivity for al1 distance ranges and that predicted by Stephens and Badwahr (1981) for $\pi^{\circ}$-decay from the demodulated spectrum of cosmic-ray nuclei.

If we assume that the electron density decreases 1 inearly with $R$ and that the electron density is effectively zero for $R^{\sim} 18 \mathrm{kpc}$ (see $\mathrm{Fig}$. 1), the radial distribution of the cosmic-ray electron density $n_{e}(R)$ and of the nuclear density $n_{n}(R)$ in the outer Galaxy is described by:

$$
\mathrm{n}_{\mathrm{e}}(\mathrm{R})=\mathrm{n}_{\mathrm{e}_{0}}\left(2.25-1.25 \frac{\mathrm{R}}{\mathrm{R}_{\theta}}\right) \text { and } \mathrm{n}_{\mathrm{n}}(\mathrm{R})=\mathrm{n}_{\mathrm{n}_{0}} \text {, }
$$

where $\mathrm{n}_{e_{0}}$ and $\mathrm{n}_{\mathrm{n}}$ are the local interstellar electron and nuclear densities respectively. The variation of the electron component is consistent with results based on low-frequency continuum surveys [e.g. at $30 \mathrm{MHz}$, Webber et az. (1980) and at $480 \mathrm{MHz}$ Phillips et az. (1981)]. 


\section{IMPLICATIONS FOR COSMIC-RAY ORIGIN}

The results presented above place constraints on the distribution of cosmic-ray sources. The strong decrease of the cosmic-ray electron density in the outer Galaxy clearly confirms its galactic origin. The interpretation of the flat nuclei distribution is beyond the scope of this paper, but a few general observations can be made.

If the sources of both species are similarly distributed in the Galaxy, extensive diffusion of the nuclei into the outer Galaxy is required to reproduce their observed flat distribution. Disk confinement will encounter difficulties in such a model and most probably a large halo would have to be invoked. However, models involving a large halo, with diffusion (Ginzburg and Syrovatskii, 1964; Ginzburg, Khazan and Ptuskin, 1980) or diffusion and convection (Owens and Jokipii 1977) generally produce gradients in the cosmic ray density on scales 3 kpc in the z-direction, and a similar scale should hold in the radial direction outside the source region. An explicit example of the distribution of cosmic rays in a diffusive halo model for the Galaxy is given in Strong (1977), and this shows a steep radial gradient. An alternative model involving Galactic origin would be a large homogeneous halo, but this is unlikely on physical grounds.

The data are consistent with an extragalactic origin for the nuclei (Brecher and Burbidge 1972, Burbidge 1974) either in its 'universal' or 'local supercluster' forms. The former is considered unlikely because of the problem of transporting the particles over intercluster distances (Burbidge 1974). The local supercluster theory avoids these problems, and has also been proposed to explain the distribution of cosmic rays above $10^{18} \mathrm{eV}$. [ see e.g. Brecher and Burbidge (1972), Burbidge (1974), Strong et al. (1974), Wdowczyk and Wolfendale (1979), Astley et al. (1981)].

L.B. gratefully acknowledges support from the Netherlands Organization for Advancement of Pure Research (ZWO), Grant No. 0407/83 from the NATO Scientific Affairs Division, and the General Research Board of the University of Maryland.

\section{REFERENCES}

Astley, S.M., Cunningham, G., Lloyd-Evans, J., Reid, R.J.0., and Watson, A.A., (1981). Proc. 17th Int. Cosmic Ray Conf. (Paris) 2, 156.

Blitz, L., Fich, M., and Stark, A.A. (1980). In Interstellar Molecules, ed. B. Andrew, Reidel, Dordrecht. p.213.

Bloemen, J.B.G.M., Blitz, L., and Hermsen, W. (1983). Astrophys. J., in press.

Bloemen, J.B.G.M., Bennett, K., Bignami, G.F., Blitz, L., Caraveo, P.A., Gottwald, M., Hermsen, W., Lebrun, F., Mayer-Hasselwander, H.A., Strong, A.W. (1984) Astron. Astrophys., submitted. Brecher, K., and Burbidge, G.R. (1972). Ap. J., 174, 253

Burbidge, G.R. (1974). Phiz. Trans. Roy. Soc. Lond. A 27?, 481.

Burger, J.J., and Swanenburg, B.N. (1971). Proc. 12th Int. Cosmic Ray Conf. (Hobart) $5,1858$.

Dodds, D., Strong, A.W., and Wolfendale, A.W. (1975). Mon.Not.R.Astr.Soc. 171, 569.

Ginzburg, V.L., and Syrovatskii, S.I. (1964). The origin of cosmic rays, Pergamon Press, Oxford Ginzburg, V.L., Khazan, Y.M., and Ptuskin, V.S. (1980). Astrophys, and Space Science 68, 295. Heiles, C., and Habing, H.J. (1974). Astron. Astrophys. Suppl. 14, 1.

Henderson, A.P., Jackson, P.D., and Kerr, F.J. (1982). Ap. J. 263, 116.

Hermsen, W. (1980). Ph.D. Thesis, University of Leiden, The Netherlands.

Issa, M.R., Riley, P.A., Strong, A.W., and Wolfendale, A.W. (1980). Nature 287, 810.

Kniffen, D.A., and Fichte1, C.E. (1981). Ap. J. 250, 389.

Kulkarni, S.R., Blitz, L., and Heiles, C. (1982). Ap. J. Letters 259, L63.

Lebrun, F., Bennett, K., Bignami, G.F., Bloemen, J.B.G.M., Buccheri, R., Caraveo, P.A., Gottwald, M., Hermsen, W., Kanbach, G., Mayer-Hasselwander, H.A., Montmerle, T., Paul, J.A., Sacco, B., Strong, A.W., Wills, R.D., and Dame, T.M., Cohen, R.S., and Thaddeus, P. (1983). Ap. J., in press.

Mayer-Hasselwander, H.A., Bennett, K., Bignami, G.F., Buccheri, R., Caraveo, P.A., Hermsen, W., Kanbach, G., Lebrun, F., Lichti, G.G., Masnou, J.L., Paul, J.A., Pinkau, K., Sacco, B., Scarsi, L., Swanenburg, B.N., and Wills, R.D. (1982). Astron. Astrophys. 105, 164.

Owens, A.J., and Jokipii, J.R. (1977). Ap. J. 215, 677.

Sacher, W., and Schönfelder, V. (1983). Galactic Astrophysics and Camma-ray Astronomy, ed.

G. Morfill and R. Buccheri, Space Sci. Rev., in press.

Stephens, S.A., and Badwahr, G.D. (1981). Astrophys. and Space Science 76, 213.

Strong, A.W., Wdowczyk, J., and Wolfendale, A.W. (1974). J. Phys. A ?, 1767.

Strong, A.W. (1977). Mon.Not.R.Astr.Soc. 181, 311.

Swanenburg, B.N., Bennett, K., Bignami, G.F., Buccheri, R., Caraveo, P.A., Hermsen, W.,

Kanbach, G., Lichti, G.G., Masnou, J.L., Mayer-Hasselwander, H.A., Paul, J.A., Sacco, B.,

Scarsi, L., and Wills, R.D. (1981). Ap. J. Letters 243, L69.

Wdowczyk, J., and Wolfendale, A.W. (1979). Proc. 16th Int. Cosmic-ray Conf. (Kyoto), $2,154$.

Weaver, H., and Williams, D.R.W. (1973). Astron. Astrophys. Suppl. 8, 1.

Williams, D.R.W. (1973). Astron. Astrophys. Supp Z. 8, 505.

Wolfendale, A.W. (1981). In Origin of cosmic rays, Reidel, Dordrecht, p.309. 